\title{
Face Recognition Based on the Key Points of High-dimensional
}

\section{Feature and Triplet Loss}

\author{
Li Zhiming \\ Center for Information Technology, Hexi University, Gansu Zhangye 734000 China
}

\begin{abstract}
Face recognition has been a hot issue in the flied of computer vision, and face recognition is increasingly applied in the actual life. However, some low dimensional features such as Gabor, LBP, SIFT couldn't achieve a good performance of face feature presentation. So an algorithm which based on the key points of high-dimensional feature is proposed. The extracted feature is transformed by Triplet Loss. The proposed algorithm firstly implement face alignment, and then extract multiscale feature. When high-dimensional features are presented, it need to be transformed by triplet loss matrix. The paper use LBP as a basic feature. Experiments results on two public three databases (LFW, PubFig) show that the propose method achieves promising results in face recognition and proves that our proposed method preforms well than the state-of-the-art single feature such as Gabor, LBP, SIFT.
\end{abstract}

Keywords: Face alignment, High-dimensional feature, Multiscale, Triplet Loss

\section{Introduction}

In recent years, face recognition technology has been widely used in mobile phone unlocking, security, payment, attendance and some other fields, meanwhile more and more demand on application prompted the rapid development of face recognition technology, thus there are more and more face recognition and research institutions and scholars. Face recognition mainly includes three parts: (I) Face preprocessing; (II) Face feature extraction; (III) Face feature matching. Among them, face feature extraction is one of the difficulties in face recognition algorithm.

A robust facial feature helps to improve the recognition rate of face recognition. At present, there are a lot of features (such as Gabor [1], LBP [2], SIFT [3]), which can be used in face recognition, they all solve the problem of facial feature to a certain extent. But these features are structurally low dimensional facial features, which can have the following disadvantages: (I) low dimensional features can show the whole facial texture features, which may ignore the details of some key parts; (II) low dimensional features can extract the characteristics of whole face, therefore some of the changes of illumination and pose can not be robust; (III) low dimensional feature can save memory, but the performance will decrease sharply when the number of recognition is increased.

So it puts forward a high dimensional feature of face recognition algorithm based on Triplet Loss, this algorithm framework includes four parts, which are as follows: (I) face image preprocessing, including face detection and face alignment; (II) feature extraction of multi-scale key points, so as to get the feature pool; (III) using the conversion features of Triplet Loss matrix to get new features of differences; (IV) matching the samples in the database so as to return the result of face recognition. 
The proposed algorithm is tested on public database LFW and PubFig, and the recognition rate is $99.8 \%$ and $95.25 \%$ respectively. The experimental result showed that the proposed method can greatly improve the face recognition rate, which can also prove the effectiveness of the proposed algorithm.

\section{The Related Research}

Face image preprocessing Face image preprocessing includes face detection and face alignment, at present, face detection algorithm has reached a bottleneck level, using the depth of learning has achieved good results. As for face alignment, such as Active Appearance Model (AAM) [4], it has achieved very good results in the face alignment, which can lay the foundation for the subsequent face recognition.

The expression of face feature Feature extraction can play an important role in face recognition. Many methods of face feature extraction have achieved good results. Reference [2] used LBP features to express the face features, which have achieved good results. However, the freedom degree of LBP features to face feature is not high. The effect is not good for the face recognition in a large scale. Reference [5] used Gabor wavelet to extract the face feature, which can be extracted to a variety of face features, but Gabor parameters are fixed, people do not know whether all of these Gabor parameters are differentiated to faces. Reference [6] adopted the depth learning method to extract face features, and the results are very good, but the depth learning needs a large amount of data to train a model, while acquiring a large amount of marks is very expensive. Reference [7][8][9] used sampling multi-scale, which was also very effective to express the face features. These methods include multi-scale LBP features, multi-scale SIFT features, which can have good effect on improving face recognition rate.

Face recognition matching Generally PCA can be used to decrease dimensionality for high dimensional data before matching face feature, using the supervised learning method, such as LDA or some metric learning method to optimize the features of extraction, however LDA only considered the similarity between the same classes, without considering the similarity between different classes, therefore there still existed great space for improvement.

The structure of this paper is as follows. In the second section, we will describe the extraction method and feature comparison of high dimensional feature. In the third section, we will introduce how to use the Triplet Loss to optimize the face feature. In the fourth section, the proposed algorithm will be tested on a public face database. The fifth section will summarize the advantages and disadvantages of the algorithm, and lay the foundation for the future work.

\section{Expression of High Dimensional Feature}

In this section, we will describe the process of extracting high-dimensional features based on the key points, as well as the influence of the number of different key points on the recognition results.

Based on the multi key points and multi-scale features extraction, the key points in this paper can be detected through the key points of human face, which are mainly distributed in eyes, eyebrows, nose, mouth and so on.The original face can be used to build up Pyramid model, then we can extract the characteristics of the key points of the face with various scales, 
because the distinction features of human mostly existed in the eyes, nose or mouth and some other key points. As long as the extraction of the characteristics of these parts can be expressed, the features of other places can be abandoned, because these are the common features of human face, which may cause interference to face recognition. Based on the extraction of high dimensional feature of the key points, five scales of human face can be used to extract the texture features of each key point. The number of key points can affect the dimension of face feature, different scales can extract different texture features, at the bottom of the Pyramid, it can extract the structural characteristics of the whole face, at the top of the Pyramid, it can extract texture features of some facial details. The features of these five scales can be combined to form a feature vector, which can be compared with the feature dictionary, so that it can get the final matching result.

As for a face in natural scene, it needs to locate the face position, then it can use AAM algorithm to detect the key points of face extraction, so as to extract high dimensional feature fir key points in the region and form the feature vector (feature vector can be got through Triplet Loss changes of PCA and the conversation of the proposed subsequent feature). The registration samples can acquire the database dictionary through feature extraction, the test samples can be compared with the database dictionary, so that it can get the final matching result.

\section{Feature Optimization of Triplet Loss}

In order to improve the performance of face recognition, a feature transform function is designed, reflecting the face feature $x$ into $R^{d}$ Euclidean space. Making the same person's European distance become smaller, making the distance between different people become larger. This kind of method is called Triplet Loss, moreover Triplet Loss can improve the face recognition rate effectively [10]. Triplet Loss is composed of a sample of three faces, the purpose of which is to make the same kind of sample set exist at the same point as much as possible, so that the characteristics of $R^{d}$ space can be existed in a better distribution, among them, $f(x) \in R^{d}$ space can be defined. It is assumed that the features are mapped to $d$-dimensional Euclidean space, taking the nearest neighbor classifier as the loss function. First of all, we should define a reference point, a negative sample point, a positive sample point, the reference point and the positive sample point is the same person's sample, as well as the reference point and the negative sample point is not the same person sample. The purpose of which is to have the face feature mapped to $R^{d}$ space, so that the distance from the reference point $x_{i}^{a}$ to the positive sample point $x_{i}^{p}$ can be closer, while the distance from the reference point $x_{i}^{a}$ to the negative sample point $x_{i}^{n}$ can be farther.

The defines loss function $\mathrm{L}$ ca be shown as follows:

$$
L=\sum_{i}^{N}\left[\left\|f\left(x_{i}^{a}\right)-f\left(x_{i}^{p}\right)\right\|_{2}^{2}-\left\|f\left(x_{i}^{a}\right)-f\left(x_{i}^{n}\right)\right\|_{2}^{2}+\alpha\right]
$$

Among $f(x)=W x$.

The above function is a convex optimization function, using the optimization method of Stochastic Gradient Descent, (SGD) [11] to get matrix W, so it needs to solve the partial derivative of $\mathrm{L}$ to $\mathrm{W}$, which can be shown as follows:

$$
\frac{\partial L}{\partial W}=\sum_{i}^{N}\left(2 W\left(x_{i}^{a}-x_{i}^{p}\right)-2 W\left(x_{i}^{a}-x_{i}^{p}\right)\right)
$$

Update $\mathrm{W}$ with each iteration:

$$
W:=W+\alpha \frac{\partial L}{\partial W}
$$


The following are steps of face recognition algorithm with high dimensional feature (KHLBP+Triplet) based on Triplet Loss:

$$
\begin{aligned}
& \text { Step1: face detection and face alignment, } \\
& \text { Step2: extraction multi scale feature for the } \\
& \text { key points of face samples, } \\
& \text { Step3: converting matrix by using training } \\
& \text { linear of Triplet Loss. }
\end{aligned}
$$

\section{Experiment and Analysis}

In this section, we will make use of the high dimension feature face recognition algorithm based on Triplet loss to carry on the experiment on the international public database. Experiments can be carried out both on LFW and PubFig face databases. The following will introduce these two databases.

LFW database consists of 5749 people with 13233 faces, each people can have face image varied from 1 to 590. All of these images can be acquired from the webpage, which can have very large class changes.

PubFig database includes 58797 face images of 200 stars, all of which can be acquired from network.

Using these data, comparing the proposed algorithm with HLBP, Gabor [5], LBP [2], SIFT [8] and some other algorithms.

\section{Experiment on LFW Database}

LFW (Labeled Face in the Wild) is face database crawling from the Internet, first of all image in LFW database should be carried out with face detection.

KHLBP + Triplet algorithm needs to learn PCA dimensionality reduction, then firstly it should get training samples, in the experiment, taking $20 \%$ of the face in database as training set, the remaining as test set. Thus face recognition experiment can be carried out in this database, finally it can get the face recognition ROC result, which can be shown in Fig.1.

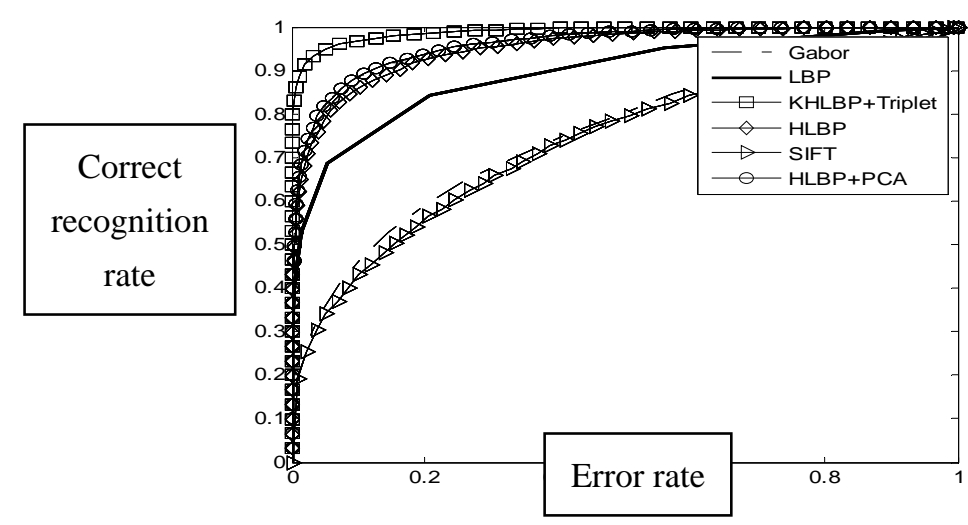

Fig.1 Experimental Result on the LFW Database

It can be seen from Fig.1, the recognition rate of the proposed KHLBP+Triplet algorithm is superior to that of the traditional algorithm such as Gabor, LBP, SIFT and so on. Thus it can be seen when the error rate is 0 , the correct recognition rate of KHLBP+Triplet algorithm can reach $85 \%$ or so. From the experimental result, we can see that the Gabor and SIFT 
features are not very good for the expression of face features, which also can prove that the proposed KHLBP+Triplet algorithm can get good result.

\section{Experiment on PubFig Database}

In the experiment, $20 \%$ of the faces in PubFig database are used as the training samples, and the remaining faces are used as the test samples for face recognition. The error rate of face recognition can be shown in Table 1.

Table 1.The Error Rate EER of Face Recognition of Different Algorithm in PubFig Database

\begin{tabular}{|c|c|c|c|c|}
\hline Method & $\begin{array}{c}\text { KHLBP+ } \\
\text { Triplet }\end{array}$ & $\begin{array}{c}\text { HLBP+P } \\
\text { CA }\end{array}$ & HLBP & LBP \\
\hline EER & $3.07 \%$ & $4.26 \%$ & $4.47 \%$ & $7.78 \%$ \\
\hline
\end{tabular}

As can be seen from Table 1, KHLBP+Triplet algorithm can achieve very good performance.

The following conclusion can be drawn from the above experiments:

1) The feature of high dimension is beneficial to the expression of face features. The higher the dimension of face feature is, the higher the recognition rate is.

2) The feature points of the extracted features from the key points are larger, because most of the features of the faces are focused on some key points of the faces.

The proposed algorithm can get good results in these public databases, it has proved that the proposed method is robust and practical, which can be used in the industrial field.

\section{Conclusion}

It proposed face recognition algorithm with high dimensional feature based on Triplet loss, first of all this algorithm can detect face, then it can position the key points of face, so as to have multi-scale texture feature extraction for each key point, lastly, having linear transformation to Euclidean feature space for these texture features by means of Triplet loss to get the distinguishing feature. This proposed algorithm can have a better performance than other algorithms in some public face databases. At the same time it can also have better effect on the expression features of high dimension of faces, which can have better robustness, using face detection to have some non-aligned face feature extraction for face recognition, which is suit for face recognition in the natural environment without restraint.

As for face recognition, there are still many aspects need to be studied, how to use deep learning to extract high-dimensional features is the direction of future research.

\section{Reference}

[1] Kong Rui, Han Yixuan. Fast face recognition algorithm based on Gabor filter [J]. Journal of Computer Applications, 2012, 32 (04): 1130-1132.

[2] Yuan Baohua, Wang Huan, Ren Mingwu. Face recognition based on complete LBP features [J]. Application Research of Computers, 2012, 29 (4): 1557-1559.

[3] Li Zhengyi, Feng Guiyu, Zhao Long. Face recognition method based on direct locality preserving projections and scale invariant feature transform [J]. Journal of Computer Applications, 2012, 32 (09): 2588-2591. 
[4] Cootes T F, Edwards G J, Taylor C J. Active appearance models [J]. IEEE Transactions on Pattern Analysis \& Machine Intelligence, 2001 (6): 681-685.

[5] Lin Jie, Li Jianping. Robust face recognition method based on Gabor wavelet transform and self adaptiion [J]. Application Research of Computers, 2008, 25 (9): 2868-2871.

[6] Taigman, Yaniv, et al. Deepface: Closing the gap to human-level performance in face verification. IEEE Conference on Computer Vision and Pattern Recognition (CVPR), 2014.

[7] Karmakar D, Murthy C A. Face Recognition using Face-Autocropping and Facial Feature Points Extraction[C]. Proceedings of the 2nd International Conference on Perception and Machine Intelligence. ACM, 2015: 116-122.

[8] Goudail F, Lange E, Iwamoto $T$, et al. Face recognition system using local autocorrelations and multiscale integration[J]. IEEE Transactions on Pattern Analysis and Machine Intelligence, 1996, 18(10): 1024-1028.

[9] Karmakar D, Murthy C A. Face Recognition using Face-Autocropping and Facial Feature Points Extraction[C]. Proceedings of the 2nd International Conference on Perception and Machine Intelligence. ACM, 2015: 116-122.

[10] G.B. Huang, M. Ramesh, T. Berg, and E. Learned-Miller. Labeled Faces in the Wild: A Database for Studying Face Recognition in Unconstrained Environments, Technical Report 07-49, Univ. of Massachusetts, Amherst, http://vis-www.cs.umass.edu/lfw/Oct. 2007. Martinez and R. Benavente, “The AR Face Database,” CVCtechnical report, 1998. 